\title{
Exercising with a Humanoid Companion is More Effective than Exercising Alone
}

\author{
Sebastian Schneider and Franz Kummert ${ }^{1}$
}

\begin{abstract}
Engineers developing socially assistive robots for promoting a healthy and active lifestyle are facing several mechanical and interactive design challenges. In this paper we are answering the question whether a humanoid robot exercising partner capable of working out together with its user makes significant improvement in the user's engagement. We conducted a study with 56 participants doing five isometric exercises in three conditions comparing the differences between working out alone (IC), with an humanoid robot instructor (RI), or with a humanoid robot companion (RC). Our results show that the RC brings a motivational gain to exercise longer compared to all other conditions.
\end{abstract}

\section{INTRODUCTION}

The attitudes that people apply towards other people have been shown to influence the way persons cooperate with others. This issue needs to be raised if we want to deploy robots as social interaction partners for rehabilitation or exercising tasks (e.g [1]). Those applications require to take a close look on how the behavior of a socially assistive robot (SAR) influences the motivation of users to adhere with a robot's instructions. For robotic design reasons, it raises the questions whether the robot needs to be able to exercise together with a trainee or if the presence and instructions of the robot are sufficient to motivate people. The research field of SAR has presented a lot of works how the presence of the robot influences the motivation and the way robots can facilitate working on a task ([1]-[3] and many more). However, no present study explicitly questions whether the mere presence is sufficient for a motivation boost or whether the robot should exercise along with the trainee. Research on the effects of group dynamics in social psychology shows that the least capable group member exhibits a relative motivation gain when performing a task in a group. This effect is called the Köhler effect, which has recently been investigated for virtual agents [4].

In this work we are pursuing two objectives: Our primary objective is to validate if this effect can also be replicated with humanoid robots.

Why is it interesting to investigate this effect with a humanoid robot? The assumption of the Köhler effect is that the motivation of a less capable team member is influenced by the presence of a more capable team member. Thus for the field of exercising, it means that the less "trained" team member will be more motivated when exercising with a more "trained" team member. The fitness level and muscle fatigue

\footnotetext{
${ }^{1}$ Sebastian Schneider and Franz Kummert are with the Applied Informatics, Faculty of Technology, CITEC, Bielefeld University, 33615 Bielefeld, Germany [sebschne, franz] @techfak. uni-bielefeld.de
}

of the more "trained" team member is however difficult to control and, for rehabilitation or exergaming tasks, an adequate training partner might not always be available. Hence, humanoid robots can be an additional tool to support physical activity, because machines have no muscle fatigue. Furthermore, regarding time scheduling problems, variability in motivation and health of a human exercising partner a humanoid robot can be a suitable addition for exercising alone and are expected to be easily available in the future.

Our secondary objective is to investigate the motivational effects of a robotic companion (RC) which is exercising together with the partner versus a robotic instructor (RI) which is only instructing the user to exercise.

We use five different abdominal isometric exericses which have already been used in previous studies to test the Köhler effect [4]. These exercises do not require high motor skills and the study design incorporates different fitness levels of persons. Thus, we might be able to reliably identify the motivational characteristics of a RC which is conjunctively exercising together with the participants compared to a RI which is solely instructing the user to do the exercises. Accordingly, we will answer the question whether the presence of a RI is sufficient for people to get motivated to exercise or if a RC is necessary which can exercise together with the user, but brings new engineering requirements for rehabilitation or exergaming tasks. We compare each of our conditions with an independent baseline condition (IC) where the participants were doing the complete workout without a robot. We hypothesize that:

H1: People exercise longer in the RC condition compared to the IC or RI condition.

$\mathrm{H} 2$ : People exercise longer in the RI condition than in the IC condition.

H3: People perceive the robot in the RC conditions as more animated, anthropomorphic, likable and intelligent than in the RI condition.

This manuscript is organized as follows: The next section gives the reader an overview of existing literature in the field of the Köhler motivation effect with virtual agents and the motivational effect of SAR for exercising. Section III introduces our study and system design. Section IV shows our obtained results and in Section $\nabla$ these results are discussed.

\section{RELATED WORK}

\section{A. Köhler Effect with Virtual Agents and Robots}

The Köhler motivation effect in sports interactions has been intensively studied by Feltz et al. [4] (for further 
references see [4]). The authors investigated different aspects of the Köhler effect to enhance training engagement and have recently reported a study where they evaluated the Köhler effect with virtual agents [4]. They have tested four different conditions (i.e. an isolated condition (IC), hardly human partner (HHP), nearly human partner (NHP), and human partner (HP)) during an isometric workout. The participants had to do 5 isometric exercises (i.e. plank, side plank left/right, plank and lifting left/right leg). Each participant had to do the exercise individually first. After the first round they had a break and then they either had a partner (HHP, NHP, HP) or did the exercises alone again (IC). Their results show that even if the participants are training together with the HHP they were holding the exercises longer than in the IC condition. Thus, the researchers could show that an artificial remote-located exercising partner is at least better than no partner at all.

Our literature review revealed one research project to study the Köhler effect with robot companions in a preliminary stage [5]. The paper does not present results or a detailed study description. we were also not able to find any followup reports on the Köhler effect with robots.

\section{B. SAR for Physical Activity}

SAR for sport assistance and physical activity have gained more attention recently [6]-[13].

Lee et al. [13] investigated the effect on motivation regarding the type of robot operation (autonomous vs. teleoperated). They conclude that a teleoperated robot increases the competition between the users and thus increases motivation to exercise compared to an autonomous robot. The limitation of this study are the choice and number of exercise (i.e. 1 exercise: holding arms in front of the body), the sample size (20 participants, within-participants design) and a missing baseline condition where no robot is present. Fasola et al. [1], [7] studied the motivational effects of a SAR in different conditions (e.g. verbal praise, relational discourse and embodiment. They investigated the positive effects of praise, relational discourse and embodiment. However, also this study is missing a baseline condition which would allow to compare the robot against the user's inherent motivation to exercise. Vircikova et al. [12] worked on the usage of Nao as an instructor for spinal disorder rehabilitation. A qualitative analysis of the humanoid-child interaction shows that the children enjoyed the exercising, had no problem to repeat the exercise and whished to continue after the rehabilitation training has finished. Görer et al. [10] developed a system that learns a set of physical exericses from a professional coach and assists people in performing these gestures. They evaluated their system in one condition with 8 participants by tracking the correctness of the gestures and a subjective questionnaire. Participants reported high scores on immersion and positive affect and low scores on flow. Werner et al. [8] studied the usage of a SAR for physical training support with older users. They evaluated the motivation after the demonstration of the training support and found that .7 of the participants think that the robot is 'very much' or 'a lot' motivating. The same amount of participants report that a human trainer would be a better motivator than the robot. However, they can not conclude on their research question to what extend the system is more motivating than a video version. Park et al. [9] investigated how social skills (i.e. mutual gaze, feedback and social distance) can enhance the social interaction in physical training in a study with two conditions (social skills vs. no social skills). They showed that such skills are effective social cues for physical training. However, no link to the effectiveness on the training engagement was made. The long-term usage of a robotic indoor cycling coach has been studied in [6], [14]. A 18-days study in an isolated environment compared the effects of an adaptive SAR giving feedback against a non-adaptive display giving no feedback. The results show that participants comply more when assisted by a robot. However, this study also lacks a baseline control group. Furthermore, the study design has two manipulation variables (embodiment and adaptive feedback). Therefore, it is not quite clear which of the variables is moderates the user's compliance. Furthermore, the study conditions were special due to a long-term isolation of the participant in a laboratory of the German Aerospace Center and also had a small sample size (8 participants for each condition).

How does our new work fill a gap in light of the presented related work? We observed that many of the works focus on light to moderate physical activity, which is mostly due to the focus of rehabilitation for children and elderly. In contrast, we are investigating the question whether a humanoid robot exercise companion is capable of pushing participants to their exercise limits in a high intensity work out. Furthermore, we are interested in the motivational effects of working out with a humanoid companion. Also, other works often focus on different types of feedback, investigate different social cues and lack a baseline comparison which shows that exercising with a robot is quantiatively excelling working out alone. Therefore, we present results from a baseline condition wich shows that working out with a humanoid companion is more motivating than working out alone.

\section{STUDY AND SYSTEM DESIGN}

The presented study design is inspired by [4]. To allow possible comparisons, we wanted to replicate the study as close as possible. However, we needed to include some changes in the study design due to the robotic agent. Therefore, we changed the exercises from forearm planks to full planks due to the robots limited degree of freedom. Also, we neglected to study differences between the embodiment of the robot. A recent meta review revealed that robots were perceived more persuasive, positive and led to higher user performance when physically present in a user's environment compared to a digitally-displayed variant of a robot or agent [15]. Furthermore, we also could not include a human partner condition, because it is impossible to control that the human experimental partner is always performing longer than the study subject, which is essential for our investigation. We could have simulated a human training partner using a fake 
video stream as in [4], but this would require to include the embodiment of the interaction partner as another source of variability. Therefore and because of the aspects mentioned earlier, we decided to exclude both a human partner condition and a virtual buddy condition. However, we still try to draw some comparisons to the results obtained from our reference study [4].

\section{A. Experimental Design and Participants}

Participants $(\mathrm{n}=56)$ were assigned to one of three conditions (independent condition (IC), robot instructor condition (RI) and robot companion condition (RC)) with 18 participants in each condition. Participants were mostly students (male: 29, female: 27 , age $\mathrm{M}=25.55$ years, $\mathrm{SD}=6.48$ ) from our university acquired by flyers distributed on the campus. They received seven Euros as monetary compensation. Three participants from the IC had to be excluded. One was an outlier already persisting much less during the first part of the session when the participants were exercising by themselves compared to all other participants. Two other persons had to be excluded because they were doing the exercises wrongly. One participant in the RI condition had to be excluded form the survey evaluation because the data were missing. In all other cases no outliers has been removed.

\section{B. Exercises and Conditions}

Participants had to do two blocks of five isometric abdominal exercises each (see Fig. 11). During the IC condition the participants did all exercises two times alone. In the other conditions the participants did the exercises alone first and with the humanoid robot $\mathrm{Naq}^{1}$ in the second block. During the RI condition the robot was announcing the exercises the user had to do, as well as how long the break is. While the users were exercising, the robot was standing in front of the user observing him/her. The robot was occasionally shifted its weight from one foot to the other while observing. After the users had finished an exercise they received a general encouraging feedback. In the RC condition the robot was uttering the same sentences as in the RI condition. But instead of just standing in front of the participant the robot was working out together with the user.

\section{System}

We modeled the interaction flow using a domain specific language for state chart description which is embedded into the framework of an event-based middle ware for distributed systems [16]. We used the NaoQI SDK version 2.1.14 for triggering text-to-speech output and motion on the robot. The exercise animations for the robot have been designed in Choregraphe. We exported this animation as Python code for a custom made tool to synchronize speech and our own designed motion on Nao. For visual and auditory perception of the user we used a Microsoft Kinect and the built-in speech recognition and face detection of NaoQI. To detect whether the user is standing or planking, we used a simple moving average on the user image of the Kinect. This

${ }^{1}$ https://www.aldebaran.com/en

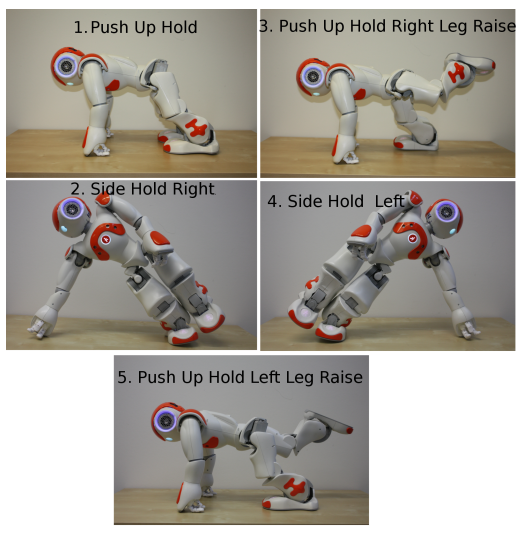

Fig. 1: The five isometric planking exercises.

component can be triggered by a remote server to calculate the current average y-position of the user in the image. Using this threshold the component can send an event to the state machine to inform about changes in the user's position. This information is also obtained by a decision server which uses an entropy decider to evaluate whether the user is still in the planking position during the movement or has finished an exercise (e.g. being below or above the y-threshold of the plank pose). While this is a fairly easy approach it was sufficient for this task. We exclude a detailed analysis of the system due to the limit of the paper.

\section{Procedure}

Participants arrived at the lab individually, read and signed a consent form which informs them that they will be recorded during the whole time of the experiment. They watched a short video of Nao demonstrating the five exercises. Afterwards, they were brought to a fitting room to change clothes and strap on a heart rate belt. They were instructed to do each exercise as long as they can and to stand up immediately if they were unable to hold the pose any longer. Then they rated their perceived exertion, took a break for 30 seconds, then continued on with their next routine. They were guided to the lab and told to start after they have waited for a short time, so that the experimenter can check that the recording is working properly. Then the participants did each exercise alone in the lab while the experimenter observed them from a different room and took the times of each exercise. The participants completed Block 1 (each exercise once). Afterwards, the participants had a ten minute break where they were offered a glass of water. After the break participants in the IC condition were told the average time they held the planks and that they would complete the same set of exercises again (Block 2 ). In every condition the participants were not told that they had to do a second block of exercises until they had finished the first block. In the RI condition participants were told that they will do the same set of exercises again but that this time a robot will be present which is going to instruct them. In the RC condition participants were also told that they will do the same set of exercises again and that this time a robot will be present. This is where the Köhler effect manipulation happend. Participants in the RC 
conditions were instructed that they would exercise as a team from now on and that the team's time is defined as the time of the person who stopped holding the plank first, which creates a conjunctive tasks. The experimenter told the participants the true average time they held the planks, like in the IC condition, but gave them a false information on how long the robot can persist the exercises. The robot's average plank time was forty percent higher than the average time of the participants, which creates an unfavorable comparison. This discrepancy, in line with previous research, leads to greater effects and was adopted from the previous study [4]. Again the experimenter did not enter the room together with the participant. Nao was waiting in the room for the participant to enter. When the system detected that a person is in the room (using the depth sensor) and standing in front of the robot (using the face detection), the robot greeted the user. In both robot conditions, the participant and robot had a short interaction phase. During this phase the robot told them its name (Nao), hometown (Paris) and hobbies (gardening, reading) and waited for a short time to give the human participant a chance to also share his/her personal information. This was done because prior research showed that people treat agents more like humans when there was an initial verbal interaction between them [17]. Following, the robot asked the user whether to start the exercises. After the robot has detected any abbrevation of 'yes' using the internal speech recognition, Nao instructed the person to go to the plank position and also went to the plank posiiton. In the RI condition the robot remained standing in front of the user, waiting for the user to go to the ground before announcing to start the first exercise. Then the robot verbally announced the next exercise and instructed to hold the exercise as long as they can. During the exercise no feedback was given to the users and the system waited until the user finished the exercise. When the participant finished the exercise by standing up or laying down the system triggered a simple motivational phrase (i.e. "you did very well"),instructed the user to rate their exertion feeling and that there will be a 30 seconds break. After the 30 seconds pause the robot announced the next exercise and the behavior repeated until the last exercise. When the participant completed the last exercise the robot thanked for the participation, told that the user is allowed to leave the room, that it needs to rest a bit and shutted itself down. After leaving the room the participant completed a questionnaire, was debriefed and received a monetary compensation. The whole procedure took about 45 minutes to one hour.

\section{E. Measures}

a) Persistence: Persistence was the number of seconds a plank was held from the moment participants moved into position until they quit. Block scores were calculated using total average seconds held on all five exercises.

b) Perceived Exertion: Perceived exertion was measured using the Borg rating scale $([18])$. The scale goes from 6 to 20 (6: "no exertion at all", 20:"maximal exertion"). The participants were asked to rate their exertion immediately

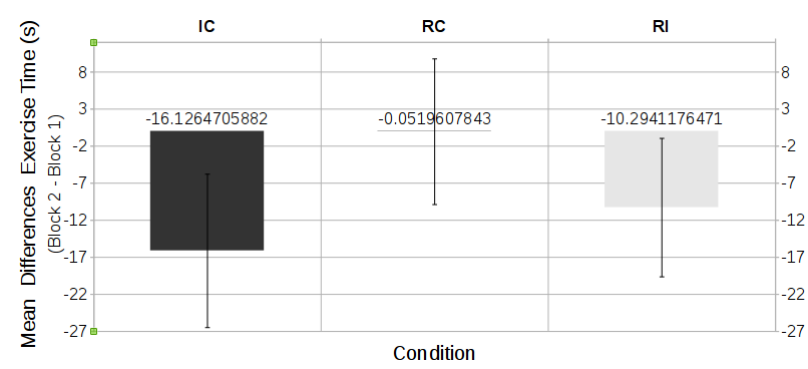

Fig. 2: Mean duration (s) exercise performance difference scores (Block 2 - Block 1) by condition.

after each exercise.

c) Godspeed Questionnaire: In order to asses different perception of the robot between the conditions we asked the participant to rate the robot based on the Godspeed questionnaire (5 point-based differential scale, [19]).

d) Role of the Interaction Partner: We asked the participants to rate which role they would ascribe to the robot using a multiple-choice input. See Figure $3 b$ for a list of available choices.

e) Physical Training Enjoyment: We assessed the physical training enjoyment of the users using the Physical Activity Enjoyment Scale ([20]). We averaged the value of all items as overall enjoyment score. Furthermore, we asked them about their intention to train tomorrow for at least 30 minutes.

f) Wish for having an assistive system: At last, we wanted to know whether participants would like to use a system that supports them during exercises in the future.

\section{RESULTS}

An analysis of variance (ANOVA) showed no differences in age or weekly physical activity between the three conditions.

\section{A. Persistence}

We adopted measurement and analysis from previous studies on the Köhler effect to be able to draw conclusions and comparisons ([4]).

A preliminary ANOVA on Block 1 persistence scores showed no significant effects. Random assignment was successful in creating no differences in the mean persistence at Block 1. This is important because a significant difference during the first block would indicate different physical fitness between the groups.

As a primary dependent variable we used the average difference persistence time in seconds between the two blocks (Block 2 - Block 1). This approach controls for individual differences in strength and fitness and shows possible changes in persistence. The results obtained for the average block score of Block 2 subtracted with the average block score of Block 1 are shown in Figure 2, A 3 (conditions) $x 1$ (persistence) ANOVA on the difference scores showed a significant main effect for the conditions $\left(F_{2,46}=11.332, p<0.001, n=.257\right)$

Participant in the IC condition persisted on average 16 seconds less at Block 2 than at Block 1. This difference 


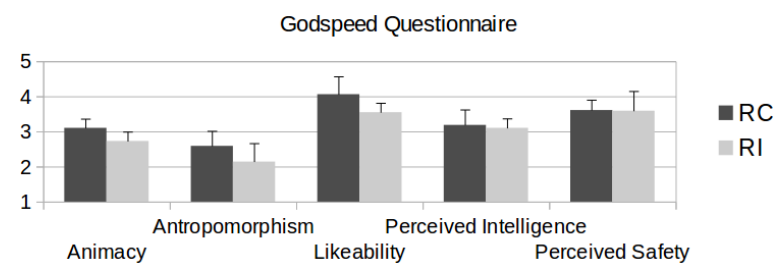

(a) Godspeed Questionnaire ratings.

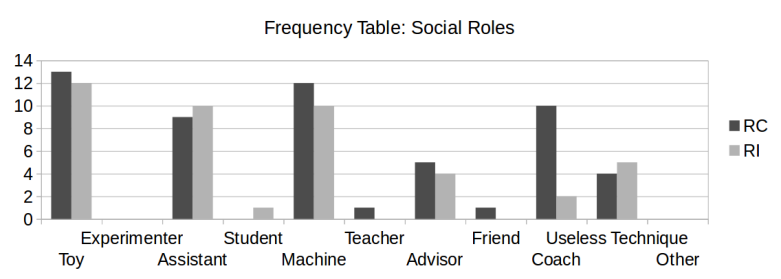

(b) Perceived social role of the system.

Fig. 3: Subjective results of this investigation.

is often observed in prior studies due to fatigue or/and boredom of the study participant [21], [22]. The results of this condition will now be used to detect any kind of motivation gain in the other conditions. Participants in the $\mathrm{RC}$ condition persisted almost the same time on average at Block 2 as in Block 1. A pairwise comparison using $t$ tests with pooled SD and Holm adjustment revealed that the difference of 16 seconds between IC and RC condition is significantly different $(p<.0001)$. Also the persistence in the RI condition was slightly greater (mean=-10.29) than the IC baseline (mean=-16.12). However, this difference is not significant $(p=.076)$ Furthermore, the persistence between the RC condition is also significantly greater than in the RI condition $(p<.01)(t(31.919)=3.1142, p<.001)$ Hence, for this participant population a difference in the usage of the embodiment of the robot did affect the magnitude of the observed Köhler effect.

\section{B. Perception of Partner}

The reliability scores of our items are: Anthropomorphism: $r=.8$, Animacy: $r=.77$, Likability: $r=.88$, Perceived Intelligence: $r=.82$, Perceived Safety: $r=0.91$. The mean and variances scores for the Godspeed questionnaire are depicted in Fig. 3a

A Welch Two Sample Test indicated that ratings for animacy from users in the RC was statistically significantly higher than in the RI $(t(31.99)=2.1313, p<.5)$. A Wilcoxon Rank Sum Test with continuity correction indicated that ratings for likeability from users in the RC were statistically significantly higher than in the RI $(Z=223.5$, $p<.01)$

\section{Perceived Exertion}

A Kruskal-Wallis Rank Sum Test (KW) for the the perceived average exertion per block showed no significant differences between the all conditions conditions $(H(2)=$ $4.451, p=.108)$.

\section{Future Assistance}

A KW for the wish for future assistance $(r=0.8)$ showed no significant differences between the conditions but a tendency $(H(2)=5.1004, p=.078)$. A post-hoc Wilcoxon Rank Sum Test showed a tendency for a difference between the IC $(\mathrm{M}=3.35, \mathrm{SD}=0.91)$ and the RI $(\mathrm{M}=2.45, \mathrm{SD}=1.23)$ condition $(p=.09)$.

\section{E. Social Role}

The frequency table for the social role ascription is depicted in Figure 3b. The frequencies between the conditions are quite similar. Nevertheless, participants in the $\mathrm{RC}$ condition perceived the system more often as a coach. There was no significant association between the kind of robot condition and the association for a social role when considering the whole frequency table using a Fisher's Exact Test for Count Data $(p=.449)$. However, there was a significant association between the type of robot condition and whether the participants ascribed the robot the role of a coach $\left(X^{2}(1)=5.6246, p<.05\right)$. This seems to represent the fact that, based on the odds ratio, the odds for the ascription of the role as a coach were $0.11(0.009,0.72)$ times higher if participants trained with the RC than with the RI.

\section{F. Ancillary Analyses}

No differences were found for the analyses of variance on intention to exercise $(P>0.11)$ and training enjoyment $(P>0.19)$ between the three conditions.

\section{G. Free Answers}

Participants in the RC condition were mostly missing motivational feedback during holding the plank, while participants in the RI condition stated that the robot should train along with them.

\section{DISCUSSION}

The primary intention of this study was to test whether the Köhler effect can be demonstrated using humanoid robotic partners in exergames. The secondary objective was to investigate whether the presence of a humanoid robotic instructor has a facilitating effect.

Our results support that performing plank exercises with a humanoid robotic partner that exercised along with the participant (RC) boosted one's effort relative to performing those exercises individually (IC). Thus, our hypothesis H1 is supported. Participants significantly increased their effort in exercising even though they were told that their partner was a robot and not a real human. This is in line with previous research where it has been found that people often respond socially to technology as if interacting with a human. Nass et al. [23] reported that people perceive computers as team mates and experience the same team dynamics similar to human teams. Still, our study is the first to validate the Köhler effect with humanoid robot partner and the 
motivatinal effects a robotic partner has when conjunctively working out with a human partner compared to working out alone or being instructed by a robot.

However, we found no support for our hypothesis $\mathrm{H} 2$ : The presence of the RI did not result in a better performance compared to the IC condition. One possible explanation can be derived from the comments of the participants. In poststudy interviews participants in the RI condition reported that they were wondering about the usefulness of the robot and that they felt irritated by its presence. In previous research on SAR supporting users in cognitive tasks we came to a similar conclusion [3]. If the usefulness of the SAR is not evident for the user, its mere presence has no facilitating effects. This conclusion contradicts other studies showing that the presence of a robot which is not explicitly exercise together with the user is also useful (i.e. [24], [25]). We have two explanations for this contradiction. First, compared to other studies, the robot in our study did not give any taskrelated feedback to the user. It announced the next exercises, reminded the user when the break is over and gave some generic encouragement after the exercises. Those utterances were neither individualized nor task-specific. Secondly, since the robot was not conjunctively exercising with the user but just present in the room and observing the user, we might have found hints for the audience effect. This effect describes that people perform better on a simple or rehearsed task in presence of others (humans and robots) and worse on complex, difficult or new tasks compared to their performance when alone [26], [27]. For now, we do not want to go into a detailed discussion about this effect due to the limited scope of this paper and simply want to point out that future investigations are needed. Those investigations could include to evaluate whether participants that are already experienced in exercising will perform better in the presence of the instructor compared to less experienced persons. Additionally, to increase the perceived usefulness of the system we will run a follow-up study where in both robot conditions ( $\mathrm{RI}$ and $\mathrm{RC}$ ) the robot is giving encouragement while exercising. Thus, we hope to distinguish whether the motivational gain is moderated by motivating encouragement or by conjunctively working out. Max et al. [28] have investigated the effects of encouragement from a human telepresent partner and trainer on the Köhler motivation effect. In all conditions they found an increase in exercise time compared to a baseline condition. However, the increase was the highest in a partner condition without encouragement. We are going to investigate whether we can replicate these results with a humanoid robot partner and instructor giving encouraging feedback while exercising.

We could find partial evidence for our hypotheses H3. The $\mathrm{RC}$ was rated higher on two of four scales of the Godspeed Questionnaire compared to the RI. It is not surprising that the $\mathrm{RC}$ was rated higher on the animacy scale. However, it is interesting that it was rated more likable than the RI due to joint training. Thus, one can hypothesize that higher ratings of likeability increase the Köhler effect for robotic exercise partners. Further research on the likeability of the robot and the relation to the motivational effects will hopefully give new insights.

At last, a significant difference in performance was measured between the conditions but no differences for exertion, intention and enjoyment were found. Participants exercised longer without any feeling to be working harder, enjoying it less or hindering their future exercise plans. These results show that it is possible to extend exercising time without negative effects. Our results are in line with the findings from Feltz et al. [4]. Their study showed that a virtual agent can increase the exercising time. Therefore, we would like to compare their result with ours regarding the question whether a virtual partner is more motivating than a humanoid exercise partner. Feltz et al. [4] showed that the persistence time was significantly higher between the IC condition and the virtual partner condition. However, the participants persisted the exercises shorter in Block 2 than in Block 1. Participants in the RC condition of our study persisted the exercises on average the same time in the second block as in the first block. Even though this is just a qualitative comparison and we have used slightly more difficult exercises in our study, one can assume that robots might be more motivating than virtual agents. Since buying a robot compared to a software program is an economical question, we propose to do more investigations in order to verify this assumption.

Compared to other studies which used robots to promote physical activity we have focused on whether co-junctively working out has an effect on the motivation to persist longer on isometric exercises. We have presented results that are comparable to a situation where the user is working solitary. So far, other works have focused on the effects of different robot behaviors, appearances or praises [7], [24], [25], but did not investigate how users would perform in an independent condition. Thus, we have presented evidence for the actual usefulness of a humanoid partner compared to having no humanoid partner.

In the following we want to discuss some limitations of our presented study. First of all, our study lacks a human partner condition. In previous research a human partner was simulated using a prerecorded video of a confederate [4]. Since we wanted to focus on the motivating effects while the partner is in the same room, we neglected a human partner condition. This decision has several reasons: The positive effects of a human partner have already been investigated. Furthermore, the implementation of a human partner condition where the human is always performing the exercises longer than the study subject is almost impossible to control since during repeated experiments at the same day it is not ensured that the human partner has no muscle fatigue and is showing always a moderately higher performance than the study subject. We could have implemented a human partner condition as a prerecorded video stream, as done in the previous researches, however then we would have introduced remote-location as another source of variability in our study design. Therefore, we concentrated our work only on the robot conditions and compared our results with previous research on computer generated training partners. 
Furthermore, the study's generalizability is limited by a static character of the isometric exercises. Therefore, we will target this issue in an upcoming iteration and let the participants do dynamic exercises (e.g. squats, push-ups, crunches). Additionally our results do not generalize to exercises or exergames which are used over an extended period of time. We will take a look on this issue and examine how the motivation changes in repeated long-term interaction with our system in a further study. This should also include how children, elderly or a clinical population could benefit from working out with a humanoid partner. Another possible extension to improve the Köhler effect is to combine cooperative play between the humanoid and human with a competition against other teams.

At last, we have recorded heart rate and video data. A closer qualitative look on the user's gaze and mimic as well as an analysis of the heart rate data might reveal new insights on the exertion and attitudes users have while working out with a robot partner.

\section{CONCLUSION}

To the best of our knowledge, this is the first study to experimentally verify the Köhler effect with a humanoid robot. It shows that exercising conjunctively with a colocated humanoid companion increases the motivation to exercise longer compared to a baseline condition and a robotic instructor. Future research on the the motivational effects of working out with a humanoid partner should examine encouragement, embodiment and likeability of the partner, the long-term effect of conjunctively exercising and a diverse study population.

\section{ACKNOWLEDGMENT}

This research was funded by grants from the Cluster of Excellence Cognitive Interaction Technology 'CITEC' (EXC 277), Bielefeld University. We want to thank our student research assistant Franziska Wäschle for her support.

\section{REFERENCES}

[1] J. Fasola et al., "A socially assistive robot exercise coach for the elderly," Journal of Human-Robot Interaction, vol. 1, no. 1, pp. 116, 2010.

[2] D. Leyzberg et al., "The physical presence of a robot tutor increases cognitive learning gains," in Proceedings of the 34th Annual Conference of the Cognitive Science Society. Austin, TX: Cognitive Science Society, 2012.

[3] S. Schneider et al., "How socially assistive robots supporting on cognitive tasks perform," in Proceedings of the 50th Anniversary Convention of the AISB, 2014.

[4] D. L. Feltz et al., "Cyber buddy is better than no buddy: a test of the köhler motivation effect in exergames," GAMES FOR HEALTH: Research, Development, and Clinical Applications, vol. 3, no. 2, pp. 98-105, 2014.

[5] K. Swift-Spong, "Studying the köhler effect with a robot exercise teammate," in Proceedings of the Human Robot Interaction Pioneers Workshop at HRI, 2013.

[6] S. Schneider et al., "Long-term feedback mechanisms for robotic assisted indoor cycling training," in Proceedings of the 3rd International Conference on Human-Agent Interaction, ser. HAI '15, Daegu, Kyungpook, Republic of Korea: ACM, 2015, pp. 157-164. DOI: $10.1145 / 2814940.2814962$
[7] J. Fasola et al., "Using socially assistive human-robot interaction to motivate physical exercise for older adults," Proceedings of the Robot and Human interactive Communication, 2012. RO-MAN 2012, IEEE, vol. 100, no. 8, pp. 2512-2526, 2012.

[8] F. Werner et al., "Evaluation of the acceptance of a social assistive robot for physical training support together with older users and domain experts," Assistive Technology: From Research to Practice: AAATE 2013, vol. 33, p. 137, 2013.

[9] C. Park et al., "A socially interactive robot using social skills in physical training," in Ubiquitous Robots and Ambient Intelligence (URAI), 2015 12th International Conference on, IEEE, 2015, pp. 437-439.

[10] B. Görer et al., "A robotic fitness coach for the elderly," in International Joint Conference on Ambient Intelligence, Springer, 2013, pp. 124-139.

[11] I. Bäck et al., "Robot-guided exercise program for the rehabilitation of older nursing home residents," Annals of Long Term Care, vol. 21 , no. 6, 2013.

[12] M. Vircikova et al., "Experience with the children-humanoid interaction in rehabilitation therapy for spinal disorders," in Robot Intelligence Technology and Applications 2012, Springer, 2013, pp. 347-357.

[13] J. Y. Lee et al., "The effect of human intervention levels of a robot on people's motivation," International Journal of Robots, Education and Art, vol. 4, no. 1, p. 9, 2014.

[14] L. Süssenbach et al., "A robot as fitness companion: towards an interactive action-based motivation model," in The 23rd IEEE International Symposium on Robot and Human Interactive Communication, IEEE, 2014, pp. 286-293.

[15] J. Li, "The benefit of being physically present: a survey of experimental works comparing copresent robots, telepresent robots and virtual agents," International Journal of Human-Computer Studies, vol. 77, pp. 23-37, 2015.

[16] A. Nordmann et al., "Modeling of Movement Control Architectures based on Motion Primitives using Domain Specific Languages," in International Conference on Automation and Robotics, 2015.

[17] T. W. Bickmore et al., "Establishing and maintaining long-term human-computer relationships," ACM Transactions on ComputerHuman Interaction (TOCHI), vol. 12, no. 2, pp. 293-327, 2005.

[18] G. Borg, Borg's perceived exertion and pain scales. Human kinetics, 1998.

[19] C. Bartneck et al., "Measurement instruments for the anthropomorphism, animacy, likeability, perceived intelligence, and perceived safety of robots," International Journal of Social Robotics, vol. 1, no. 1, pp. 71-81, 2009.

[20] D. Kendzierski et al., "Physical activity enjoyment scale: two validation studies.," Journal of Sport \& Exercise Psychology, 1991.

[21] N. L. Kerr et al., "The köhler group motivation gain: how to motivate the weak links in a group," Social and Personality Psychology Compass, vol. 5, no. 1, pp. 43-55, 2011.

[22] B. C. Irwin et al., "Aerobic exercise is promoted when individual performance affects the group: a test of the kohler motivation gain effect," Annals of Behavioral Medicine, vol. 44, no. 2, pp. 151-159, 2012.

[23] C. Nass et al., "Can computers be teammates?" International Journal of Human-Computer Studies, vol. 45, no. 6, pp. 669-678, 1996.

[24] A. Powers et al., "Matching robot appearance and behavior to tasks to improve human-robot cooperation," Human-Computer Interaction Institute, p. 105, 2003.

[25] A. Tapus et al., "User robot personality matching and assistive robot behavior adaptation for post-stroke rehabilitation therapy," Intelligent Service Robotics, vol. 1, no. 2, pp. 169-183, 2008.

[26] B. Strauss, "Social facilitation in motor tasks: a review of research and theory," Psychology of sport and exercise, vol. 3, no. 3, pp. 237256, 2002.

[27] N. Riether et al., "Social facilitation with social robots?" In HumanRobot Interaction (HRI), 2012 7th ACM/IEEE International Conference on, IEEE, 2012, pp. 41-47.

[28] E. Max et al., "Is silence really golden? effect of encouragement from a partner or trainer on active video game play," International Journal of Sport and Exercise Psychology, pp. 1-15, 2016. 